\title{
CORRECTION
}

\section{Correction to: CLIC1 knockout inhibits invasion and migration of gastric cancer by upregulating AMOT-p130 expression}

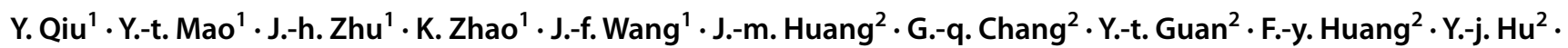
J.-q. Chen ${ }^{1} \cdot$ J.-I. Liu' ${ }^{1}$

Published online: 16 September 2020

(c) Federación de Sociedades Españolas de Oncología (FESEO) 2020

\section{Correction to: Clinical and Translational Oncology https://doi.org/10.1007/s12094-020-02445-0}

In Figure 5B, the representative image of the KD-NC group was mistakenly uploaded. The authors apologize for any confusion this may have caused. The correct Fig. 5 is given below.

The original article can be found online at https://doi.org/10.1007/ s12094-020-02445-0.

J.-q. Chen

gxhans@163.com

$\triangle$ J.-1. Liu

liujinlugx@163.com

1 Department of Gastrointestinal Surgery, The First Affiliated Hospital of Guangxi Medical University, 6 Shuangyong Road, Nanning 530021,

Guangxi Zhuang Autonomous Region,

People's Republic of China

2 The Guangxi Medical University, Nanning, Guangxi Zhuang Autonomous Region, People's Republic of China 

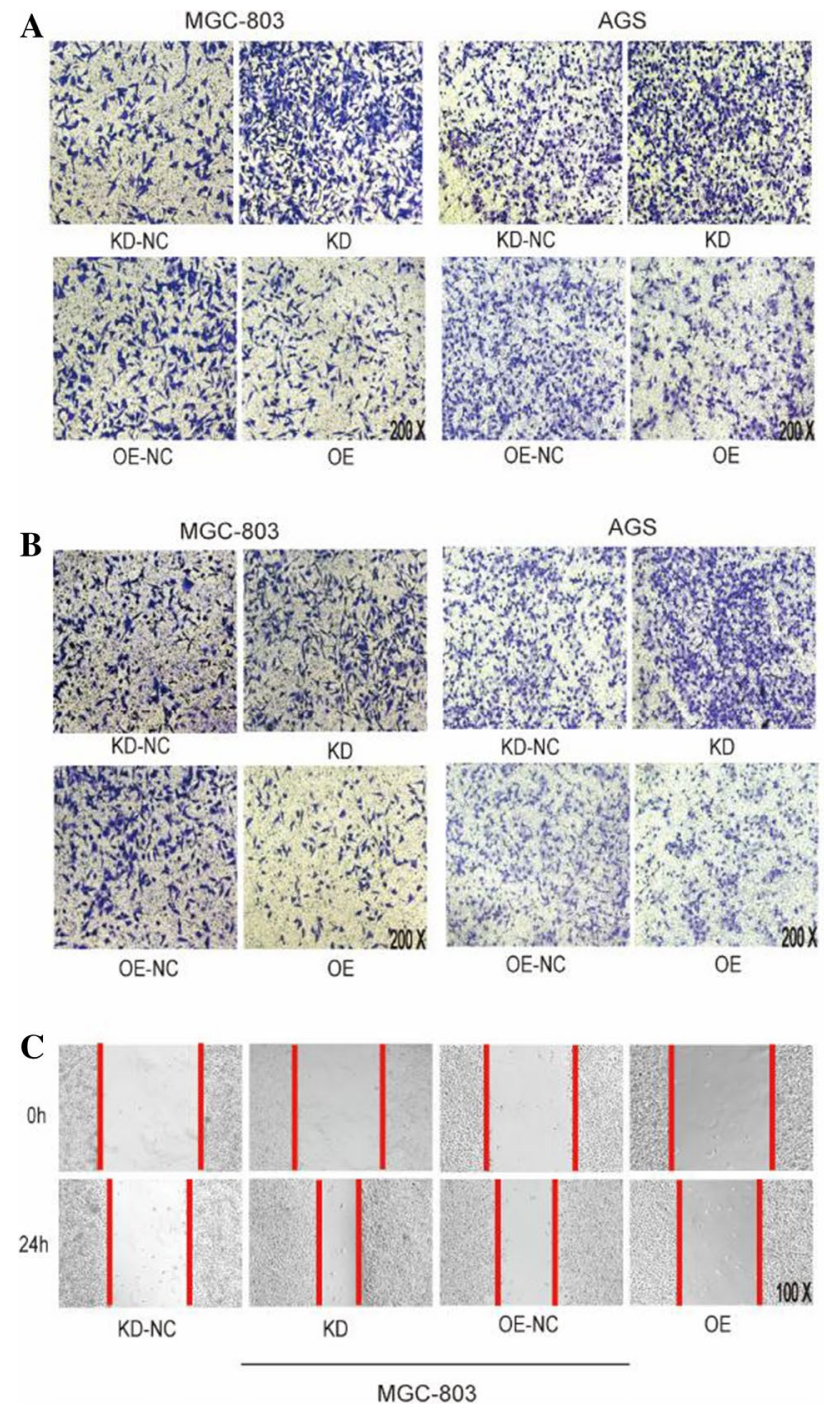

Fig. 5 AMOT-p130 inhibits invasion and migration of GC cells. a The cell migration assay showed that the number of cells passing through the membrane in the $\mathrm{KD}$ group was significantly higher than that in the KD-NC group of cells $(P<0.05)$, while the number of cells crossing through the membrane in the OE group was less than the OE-NC group of cells $(P<0.05)$. b The cell invasion assay

Publisher's Note Springer Nature remains neutral with regard to jurisdictional claims in published maps and institutional affiliations.
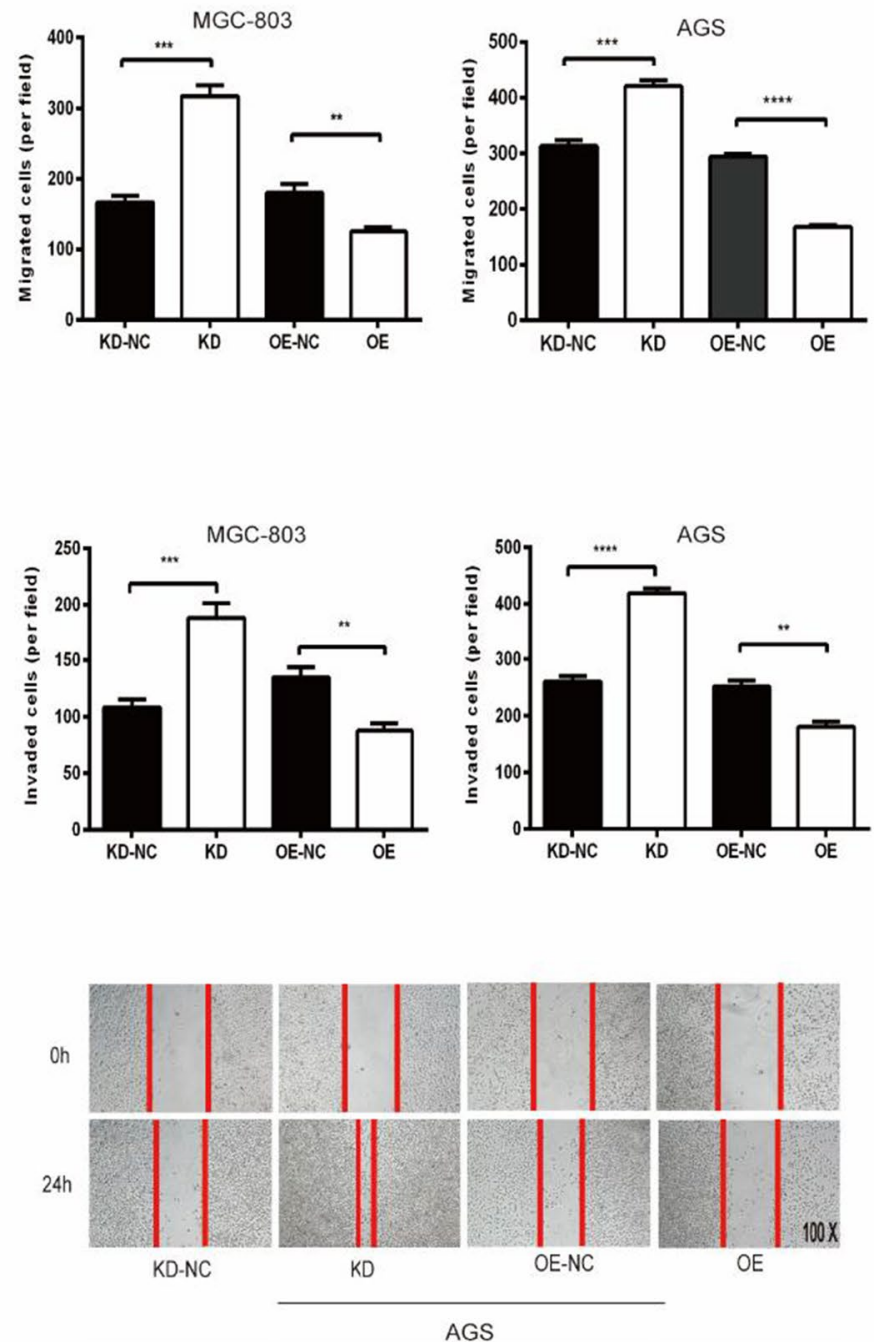

showed that the number of cells invaded in the KD group was higher than the KD-NC group $(P<0.05)$, while the $\mathrm{OE}$ group was decreased compared with the OE-NC group $(P<0.05)$. c The recovery of cells in the KD group was significantly faster than the KD-NC group. The recovery of cells in the OE group was slower than the OE-NC group 